\title{
Software Design for Particles in Incompressible Flow, non-subcycled case
}

\author{
Dan Martin and Phil Colella \\ Applied Numerical Algorithms Group *
}

February 8, 2005

\section{Overview}

To implement an AMR incompressible Navier-Stokes with particles algorithm, we have decided to use a non-subcycled algorithm to simplify the implementation of the particle drag forcing term. This requires a fairly broad redesign of the software from what was presented in [1], since we will no longer be using the AMR/AMRLevel base classes to manage the AMR hierarchy. The new classes map on to the functionality of the classes in the original design in a fairly straightforward way, as illustrated in Table 1. The new PAmrNS class takes on the functionality of the ParticleAMRNS class in the earlier implementation, along with the functionality of the AMR and AmrLevel classes in the Chombo AMRTimeDependent library. The new AmrProjector class replaces the original CCProjector class, while the new AMRParticleProjector class replaces the original ParticleProjector class.

A basic diagram of the class relationships between the AMRINS-particles classes is depicted in Figure 1.

The PAmrNS class will manage the AMR hierarchy and the non-subcycled advance. The non-subcycled advance is much simpler than the subcycled case, both in terms of algorithmic complexity (no need for synchronization projections, etc) and in terms of software implementation.

${ }^{*}$ This work supported by the NASA Earth and Space Sciences Computational Technologies Program and by the U.S. Department of Energy: Director, Office of Science, Office of Advanced Scientific Computing Research under Contract DE-AC02-05CH11231. 


\begin{tabular}{|c|c|}
\hline Old Implementation & New Implementation \\
\hline \hline ParticleAMRNS & PAmrNS \\
AMR & \\
AmrLevel & AmrProjector \\
\hline CCProjector & AMRParticleProjector \\
\hline ParticleProjector &
\end{tabular}

Table 1: Mapping of functionality of classes in new implementation to those in the original Navier-Stokes with particles implementation.

\begin{tabular}{|c|c|}
\hline PAmrNS & \\
\hline \multirow{4}{*}{$\begin{array}{l}\text { m_projector: class AmrProjector } \\
\text { m_particleProj: class AMRParticleProjector } \\
\text { u_old, u_new: Vector }<\text { LevelData }<\text { FArrayBox }>> \\
\text { m_particles: Vector }<\text { LevelData }<\text { BinFab }<\text { DragParticle }>>\text { > }\end{array}$} & \\
\hline & AmrProjector \\
\hline & m_vectGrids: Vector $<$ DisjointBoxLayout $>$ \\
\hline & \multirow{5}{*}{$\begin{array}{l}\text { MacProject() } \\
\text { CCProject() } \\
\operatorname{gradPhi}() \\
\operatorname{gradPi}() \\
\text { initialVelocityProject() }\end{array}$} \\
\hline $\operatorname{doRun}()$ & \\
\hline advance () & \\
\hline computeAdvectionVelocities() & \\
\hline predictVelocities() & \\
\hline \multirow{5}{*}{$\begin{array}{l}\text { postTimeStep() } \\
\text { regrid() } \\
\text { postRegrid() } \\
\text { initializePressure() } \\
\text { initialData() } \\
\text { postInitialize() } \\
\text { computeDt() } \\
\text { computeInitialDt() }\end{array}$} & \\
\hline & \\
\hline & AMRParticleProjector \\
\hline & m_vectGrids: Vector $<$ DisjointBoxLayout $>$ \\
\hline & projectForce() \\
\hline
\end{tabular}

Figure 1: Software configuration diagram for the AMRINS particle code showing basic relationships between AMRINS-particle code classes 
The AMRParticleProjector will do the particle projection originally implemented in the ParticleProjector class on an AMR hierarchy, including all image particle effects. The rest of the implementation (DragParticle, etc) will be the same as in the original software design.

Since much of the functionality and internal storage in the CCProjector class in the original AMRINS code is devoted to subcycling-related functionality, the AmrCCProjector is a stripped-down version of the CCProjector which only contains the functionality needed to do the multilevel cell-centered and face-centered projections.

\section{Class Outline}

\subsection{The AMRParticleProjector class}

The AMRParticleProjector class encapsulates the functionality needed to take the individual forces in the particles and apply them to the mesh in an approximation to $\mathcal{P} \vec{f}$, which may then be used as a source term for the Navier-Stokes advance. Note that the particles and projected force may be on different grid hierarchies; in parallel this allows the use of different grid

distributions which are load balanced for particles and for the fluid (the force will generally be on the same grids as the fluid).

Public Functions:

- void define(const Vector<DisjointBoxLayout $>\&$ a_vectGrids, const Vector<DisjointBoxLayout $>\&$ a_vectParticleGrids, const Vector<ProblemDomain $>\&$ a_vectDomain, const Vector<int>\& a_vectRefRatio, const Vector $<$ Real $>\&$ a_vectDx, const Vector<LevelData<BinFab<DragParticle> $>*>\&$ a_vectParticles Real a_spreadingRadius, Real a_correctionRadius $=2$, int a_gridsGrow=0);

Defines class object.

- a_vectGrids - grid hierarchy for projected particle drag force.

- a_vectParticleGrids - grid hierarchy which contains particles.

- a_vectDomain - Problem domains for grid hierarchy. 
- a_vectRefRatio - refinement ratios.

- a_vectDx - cell spacings.

- a_vectParticles - current particles (needed to generate grown grids).

- a_spreadingRadius

- a_correctionRadius

- a_gridsGrow - amount of padding to add to grown grids to account for particle motion between regridding steps.

- void projectForce(Vector<LevelData $<$ FArrayBox $>*>\&$ a_force, const Vector<LevelData<BinFab<DragParticle $>>*>\&$ a_particl

Given the collection of DragParticles in a_particles, returns the projection of the force at cell centers in a_force, suitable for use as a source term for the INS advance.

- void setSpreadingRadius (const Real a_rad) Sets spreading radius for MLC part of algorithm

- void setCorrectionRadius(const Real a_rad) Sets correction radius for MLC part of algorithm.

Protected Functions:

- void computeD(Vector<LevelData<FArrayBox $>*>\&$ a_D, const LevelData<BinFab<DragParticle $>>*>\&$ a_particles)

- void solveForProjForce(Vector<LevelData $<$ FArrayBox $>*>\&$ a_projectedForce, const Vector<LevelData<FArrayBox $>*>\&$ a_D, int a_lbase);

- void defineImages (List<DragParticle $>\&$ a_imageParticles, const Vector<LevelData<BinFab<DragParticle $>>*>\&$ a_particl

void defineGrownGrids (const Vector<DisjointBoxLayout>\& a_grids, const Vector $<$ LevelData<BinFab $<$ DragParticle $>>*>\&$ a_vec 


\subsection{The PAmrNS class}

This PAmrNS class manages the AMR hierarchy and the non-subcycled advance of the solution. In terms of functionality, it combines the functionality of the AMR and AMRLevel base classes, but with much simplified functionality due to the non-subcycled advance.

Public Functions:

- PAmrNS (const ProblemDomain\& a_baseDomain, const Vector<int>\& a_vectRefRatio, const RealVect\& a_domainLength, int a_maxLevel)

Full constructor (calls matching define function).

- a_baseDomain - problem domain for the coarsest level in the AMR hierarchy

- a_vectRefRatio - refinement ratios for the AMR hierarchy

- a_domainLength - size of the physical domain.

- a_maxLevel - finest allowable level in the AMR hierarchy

- void setUpForFixedHiearchyRun(Vector<Vector<Box > > a_vectGrids)

Set up grids, initialize data, etc for fixed hierachy run

- a_vectGrids - boxes for AMR grid hierarchy

- void setUpForAmrRun()

Set up grids, initialize data, etc for AMR run

- void PAmrNS()

destructor

- Real doRun(Real a_maxTime, int a_maxStep) advance solution - returns time of final solution.

- a_maxTime - maximum time to advance solution to

- a_maxStep - maximum number of steps to advance solution 
- int writeCheckpointFile(const string\& a_fname)

writes checkpoint file suitable for restarting. Returns status code (0 $==\mathrm{OK})$.

- int writeCheckpointFile(HDF5Handle\& a_handle) writes checkpoint file for restarting

- int writePlotFile() write plotfile using default filename construction.

- int writePlotFileName(const string\& a_fname)

- int writePlotFileHDF5(HDF5Handle\& a_handle)

Protected Functions:

- Real advance(Real a_dt);

- void postTimeStep();

- void tagCells(Vector<IntVectSet>\& a_tags);

- void tagCellsLevel(IntVectSet\& a_tags);

- void tagCellsInit(IntVectSet\& a_tags);

- void regrid(const Vector<Vector<Box> >\& a_new_grids);

- void postRegrid();

- void initialGrid(const Vector<Box $>$ \& a_new_grids);

- void initialData();

- void postInitialize();

- void writeParticleData(HDF5Handle\& a_handle) const;

- int writeParticlesHDF5(HDF5Handle\& a_handle, const List<DragParticle $>$ \& a_particles) const;

- Real computeDt(); 
- Real computeInitialDt();

- void CFL(Real a_cfl);

- void particleCFL(Real a_particle_cfl);

- void refinementThreshold(Real a_refine_threshold);

- void limitSolverCoarsening(bool a_limitSolverCoarsening);

- int finestLevel() const;

- int maxLevel() const;

- bool isEmpty(a_level) const;

- void computeVorticity(LevelData $\langle$ FArrayBox $>\&$ a_vorticity, int a_level) const;

- void computeKineticEnergy(LevelData<FArrayBox $>$ \& a_energy, int a_level) const;

- Real particleKineticEnergy() const;

- void dumpParticlesASCII(std: ostream\& os ) const;

\subsection{The AmrProjector class}

The AmrProjector class manages the enforcement of the divergence constraint for both cell-centered and face-centered (MAC) velocities. The class also differs from the original CCProjector class in that it owns no data itself. This simplifies its design considerably.

Public Functions:

- AmrProjector (const Vector<DisjointBoxLayout>\& a_vectGrids, const Vector<ProblemDomain $>\&$ a_vectDomain, const Vector $<$ Real $>\&$ a_vectDx, const Vector<int>\& a_vectRefRatio, int a_lbase, const PhysBCUtil\& a_physBC)

Full constructor - calls matching define function.

- a_vectGrids - AMR grid hierachy 
- a_vectDomain - problem domains

- a_vectDx - cell spacings for the AMR hierarchy

- a_vectRefRatio - refinement ratios

- a_lbase - base level

- a_physBC - object containing physical BC info.

- void regrid(const Vector<DisjointBoxLayout>\& a_newGrids)

Redefine grid hierarchy for projection after regridding

- void init(const AmrProjector\& a_oldProj)

Initialize new projection with data from old projection

- void MacProject(Vector<LevelData<FluxBox>* > \& a_uEdge, Vector $<$ LevelData $<$ FArrayBox $>*>\&$ a_vectPhi, Real a_oldTime, Real a_dt)

Do a multilevel face-centered projection of uEdge.

- a_uEdge - face-centered velocities.

- a_vectPhi - correction field generated by the projection.

- a_oldTime - time at the beginning of the timestep.

- a_dt - timestep used for advance.

void CCProject(Vector<LevelData<FArrayBox>* >\& a_velocity, Vector $<$ LevelData $<$ FArrayBox $>*>\&$ a_vectPi, const Real a_newTime, const Real a_dt)

Performs a multilevel cell-centered projection of a_velocity.

- a_velocity - cell-centered velocity field.

- a_vectPi - correction field (pressure) generated by the projection.

- a_newTime - time at the end of the timestep.

- a_dt - timestep.

- void initialVelocityProject(Vector<LevelData<FArrayBox>* >\& a_velocity) Project a_velocity using a multilevel cell-centered projection. Differs from CCProject in that a correction is not returned. 
- void gradPhi(LevelData<FArrayBox $>$ \& a_gradPhi, const Vector $<$ LevelData<FArrayBox $>*>\&$ a_vectPhi, int a_dir, int a_level) const

Returns face-centered $\operatorname{grad}(\mathrm{phi})$ in direction dir.

- a_gradPhi - dir-component of face-centered Grad(phi).

- a_vectPhi

- a_dir - component of gradient to compute

- a_level - which AMR level on which to compute grad(phi)

- void gradPhi (LevelData $<$ FluxBox $>$ \& a_gradPhi, const Vector<LevelData<FArrayBox $>*>\&$ a_vectPhi, int a_level) const

Returns all components of grad(phi) (gradPhi should be correct size). This includes transverse components.

- a_gradPhi - face-centered grad(phi), which should have SpaceDim components on each face.

- a_vectPhi

- a_level - which AMR level on which to compute grad(phi)

- void gradPi(LevelData<FArrayBox>\& a_gradPi, const Vector<LevelData<FArrayBox>* >\& a_vectPi, int a_dir, int a_level) const

Computes grad(pi) in direction dir (similar to gradPhi function).

- void gradPi(LevelData<FArrayBox>\& a_gradPi, const Vector<LevelData<FArrayBox>* >\& a_vectPi, int a_level) const

returns $\operatorname{grad}(\mathrm{pi})$ in all directions into SpaceDim-dimensioned gradPi.

\section{References}

[1] Dan Martin and Phil Colella. Software Design for Particles in Incompressible Flow. Applied Numerical Algorithms Group, Lawrence Berkeley Laboratory, 2003. 